\title{
Robust Multi-Rate Modulation for Cognitive Radio Communications over Land Mobile Satellite Channel at Ku-Band
}

\author{
Maurizio Murroni ${ }^{1 *}$ \\ email: murroni@diee.unica.it \\ *Corresponding author \\ Vlad Popescu \\ email: vlad.popescu@unitbv.ro \\ Mauro Fadda ${ }^{3}$ \\ email: mauro.fadda@cnit.it \\ Daniele Giusto ${ }^{1}$ \\ email: $\underline{\text { ddgiusto@unica.it }}$
}

\author{
1- DIEE, University of Cagliari, Piazza d'Armi, 09123 Cagliari, Italy \\ 2- DEC, Transilvania University of Brașov, 500036, Braşov, Romania \\ 3-CNIT, Cagliari Research Unit, P.zza d'Armi 09123, Cagliari, Italy
}

\begin{abstract}
The usable satellite spectrum is becoming scarce due to continuously increasing demand for broadcast, multimedia and interactive services. Due to the extensive use of predictive coding, multimedia data exhibit different sensitivities to transmission errors, which makes them suitable for unequal error protection (UEP) techniques. UEP mainly relies on differentiated error control coding which raises the robustness of data, but also increases the transmission bandwidth, which can be unwanted in case of limited resources often occurring in satellite communications. In this context, cognitive radio (CR) satellite communications has received important attention lately in the research community. In multi-rate modulations (MRM) the properties of dy-homogeneous signals are exploited to transmit over multiple band frequencies and time scales. MRM have been proved to be effective on unknown channels either in bandwidth or time. These conditions occur in CR networks where opportunistic communications are set up while preserving primaries licensed users. In CR the uncertainty of transmission opportunity makes the use of error correcting codes not trivial. Therefore, to achieve robustness this work proposes unequal power allocation (UPA) over the symbols of MRM according to both data sensitivity to channel errors and spectrum availability. As optimization criterion the mean square error between the original and the decoded message, which has showed strong correlation with subjective perception, is used. To assess the effectiveness of the proposed scheme in realistic condition for CR satellite networks the Land Mobile Satellite (LMS) model at Kuband is used to reproduce the memory associated with real channels. The non-linearity of the satellite channel due to high power amplifier (HPA) prior to transmission has been accounted using the Saleh model.
\end{abstract}


Keywords: Multi-Rate Modulation, Cognitive Radio Networks, Unequal Error Protection, Modulation with Unequal Power Allocation, Land Mobile Satellite Model. 


\section{INTRODUCTION}

Until the recent past years, satellite transmission at Ku-band (10-12 GHz) was traditionally dedicated to stationary services. This was mainly due to the potential interference among neighbor satellites and to the need of high-gain directive antennas at the mobile receiver which requires satellite tracking capabilities [1]. However, the relative high number of existing $\mathrm{Ku}$-band transponders and the important amount of available bandwidth has made it attractive for several operators and researchers of the satellite communication fields. Modern satellite communications more and more deal with transmission of digital multimedia compressed streams. The objective is to provide broadband interactive services to mobile users located on aircrafts, boats, and vehicles such as high speed trains, busses, and cars [2]. Due to the extensive use of predictive and variable length codes, a compressed stream is in general more vulnerable to data losses and transmission errors, which can desynchronize the decoder causing spatial and temporal error propagation. Unequal error protection (UEP) is a channel coding technique used to increase the robustness of data which exhibit different sensitivities to transmission errors. Since, in satellite transmission feedback channel is rarely available, UEP relies on differentiated forward error correction (FEC) coding.

The demand for broadband wireless spectrum is increasing due to a rapidly expanding market of high speed broadband and multimedia wireless services while the usable spectrum is becoming scarce due to current spectrum segmentation and static frequency allocation policies. In order to fully exploit the spectrum resources, in Cognitive Radio (CR) networks secondary users aim at transmitting in licensed bands avoiding harmful interference to primaries [3]. Extensively investigated for terrestrial wireless communications in the last fifteen years [4], recently CR paradigm has been considered also for satellite communications [5]. In CR, a huge number of autonomous communications shares the spectrum and the a priori knowledge of available bandwidth $B$ or time $T$ is denied due to their unpredictable fluctuations. Adaptive complex schemes are adopted to sense the channel and transmit if opportunities are available. Power measurement [6], statistic [7], cooperative [8] and multiband [9] approaches have been proposed to identify the spectrum holes potential candidate for opportunistic transmission. A transmission opportunity is defined by the product $B \times T$, when $B$ and $T$ are not simultaneously known. The uncertainty of both $B$ and $T$ makes channel coding not trivial in CR networks and can dramatically increase the complexity of the communication systems.

Multi-rate modulations (MRM) [10] have been proposed by Wornell and Oppenheim for transmission over unknown channels in frequency or time, but with fixed values of $B \times T$, which are the typical conditions of CR communications. MRM are based on dy-homogeneous signals which allow transmitting over multiple band frequencies and time scales and for that reason were also referred as Wavelet or Fractal Modulation. MRM were extensively studied over AWGN and fading wireless channels [11].

In literature, in case of digital wireless communication systems not including for some reason (e.g., high complexity design, bandwidth constraint, etc.) channel coding, Modulation with Unequal Power Allocation (MUPA) [12] has been proposed as alternative approach to channel coding to increase resilience in conventional modulation schemes. Considering that errors on the Most Significant Bit (MSB) affect the transmission more than faults on the Least Significant Bit (LSB), MUPA achieves unequal error protection by distributing the power, according to the data sensitivity to channel error. MUPA was investigated for several uncoded modulation schemes in presence of AWGN [12] and fading channels [13].

In [14] a MUPA scheme for MRM for CR networks has been proposed to improve transmission. The power budget was allocated to both optimize the quality of the received data and avoid interference with primary users. The system was tested over AWGN, which does not realistically reproduce the behavior of the wireless channel in CR networks where the hidden node margin issue due to shadowing and fading effects can severely impair the communication [1]. Furthermore, in [14] the non-linearity issues typical of the satellite transmission chain have not been addressed.

In this work, the performance of the MUPA scheme applied to MRM in realistic condition for CR satellite networks is analyzed. Assuming perfect knowledge of the channel opportunities, that is considering spectrum 
sensing reliable results available, different typical CR transmission scenarios have been considered by varying channel opportunity (i.e., $B \times T$ ) availability from low to high busy channel. Furthermore, to reproduce the memory associated with the LMS channel the two state Markov model has been used at Kuband. LMS is a first-order Markov chain that has been used in literature to model impairment introduced by narrowband fading/shadowing in case of digital satellite communications [15]. The non-linearity of the satellite channel due to high power amplifier (HPA) prior to transmission has been accounted using the Saleh model [16].

As in [14], in this work the optimization criterion for the unequal power allocation is the minimum Mean Square Error (MSE) between original and received data that has showed during the tests strong correlation with subjective perception [12][13][14].

Due to the complexity of the optimization problem, a numerical solution to the power weight optimization using Genetic Algorithms (GA) [17] has been used. In computer science GA are search techniques used to find iterated approximate solution to optimization problems with an accuracy dependent on iterations. Solutions based on GA have been proposed in communication engineering [18] [19] [20] [21]. In the proposed system, the optimization is performed off-line under the hypothesis of perfect knowledge of the channel opportunity $B \times T$. Known the $B \times T$ constraint, the proposed system will implement opportune unequal power distribution on the available carriers according to a look up table strategy. Therefore, no real time nor convergence time issues have been addressed in this work.

Extended tests have shown that the proposed scheme allows MRM to increase resilience of data that exhibit different sensitivities to channel errors for transmission in CR network under realistic operation conditions. The quality improvement in the parameter domain (i.e., MSE) has been proved against an equally distributed MRM system, in the presence of similar $B \times T$ constraint. Furthermore, transmission of real signals allowed validating the correspondence between quality achieved in the MSE domain and subjective perception. Moreover, since in satellite CR resource allocation is a key issue due to high costs, the bandwidth saving obtained at fixed quality (i.e., fixed MSE) at receiver has been evaluated to show the MUPA increased performance compared to conventional UEP based on FEC coding.

In the rest of the paper sections 2 and 3 describe the MRM and the LMS channel at Ku-band, respectively. The communication system is presented in section 4 , whereas the power optimization is described in section 5. Test and results are presented in section 6. Finally, section 7 draws the conclusion.

\section{Multi-RATE MODULATION}

MRM uses dy-homogenous signals to transmit over multiple frequency bands and time scales. Given orthonormal wavelet basis, $\psi_{n}^{m}=2^{m / 2} \psi\left(2^{m} t-n\right)$, with $m$ and $n$ dilation and translation indices, respectively, $s(t)=\sum_{m} \sum_{n} \beta^{m / 2} q[n] \psi_{n}^{m}(t)$ where $q[n] \in\left\{ \pm \sqrt{E_{b}}\right\}$, being $E_{b}$ bit energy and $\beta=2^{2 H+1}$, is able to carry data at multiple rates. The time - frequency portrait is shown in figure 1 for MRM. The durationbandwidth product $B \times T$ limit the coefficients that can be accessed. A band-limit of $2^{T} U \mathrm{~Hz}$ for some integer $T_{U}$, the denies access to the coefficients at scales $m>T_{U}$. Similarly, a time-constraint produces a minimum allowable decoding rate of $2^{T_{L}}$ symbols/sec for some integer $T_{L}$, which limit the access to the coefficients at scales $m<T_{L}$. The error-probability depends on both rate $R$ and bandwidth $B$ [10]:

$P_{r}(\varepsilon)=Q\left(\frac{1}{2} \sqrt{\gamma_{b}\left[\frac{2 \eta_{F}}{R / B}-1\right]}\right)$

where $\eta_{F} \approx 1 / 2$ and $\gamma_{b}$ is the SNR. Figure 2 shows modulator and demodulator for MRM. 


\section{THE LMS CHANNEL MODEL AT KU-BAND}

At data rate of practical interest (up to several tens of Mbps) the LMS channel model at Ku-band is non frequency selective, large scale fades are normally modelled by means of a Markov chain which determines the transitions among channel states, whereas the small scale fades within each state are modelled by means of suitable statistical distributions, such as Rice, Rayleigh, etc. [1]. A simplified, but extensively adopted model for the LMS channel is the Lutz's model [15] in which a two-state good and bad, namely nonshadowing and shadowing state, model is defined. In the non-shadowing state, the received signal amplitude $r$, follows a Rician distribution, $p_{g o o d}=2 k r e^{-k\left(r^{2}+1\right)} I_{0}(2 k r)$ where $k$ is a Rice factor and $I_{0}$ the zeroorder Bessel function. In the shadowing state, no direct signal path exists and the multipath fading has a Rayleigh characteristic with its envelope $s_{0}$ following a lognormal distribution. The probability density functions (pdfs) of the multipath fading and its envelope are $p_{b a d}\left(r / s_{0}\right)=\left(2 r / s_{0}\right) e^{-\left(r^{2} / s_{0}\right)}$ and $f_{l g}\left(s_{0}\right)=$ $\frac{10}{s_{0} \sqrt{2 \pi} \sigma \ln 10} e^{-\left(10 \log s_{0}-\mu^{2}\right)^{2} / 2 \sigma^{2}}$ respectively. In the bad state, the pdf of the channel is actually a Suzuki distribution $p_{b a d}(r)=\int_{0}^{\infty} p_{b a d}\left(r / s_{0}\right) f_{l g}\left(s_{0}\right) d s_{0}$. The two states good and bad are time-sharing and can be modelled as a second order Markov model. Assuming that $A$ is the average duration of the bad state, the pdf of the received signal amplitude can be represented by

$p_{L S M}(r)=(1-A) p_{\text {good }}(r)+A p_{\text {bad }}(r)$

\section{SYSTEM MODEL}

In $\mathrm{CR}$ scenario the channel opportunities (i.e., $B \times T$ ) can be identified by spectrum sensing to be periodically performed before transmission [6]. In the proposed system, we assume perfect knowledge of the transmission opportunities and MRM is designed to exploit the available transmission opportunities: if the channel constraints do not allow the transmission of the signal at all the resolutions, corresponding wavelets carriers which can be potentially harmful are suppressed. Once constellation symbols are modulated, they are amplified by the high power amplifier (HPA) prior to transmission, thus being subject to the non-linear behaviour of the amplifier, whose effects can be modeled using the Saleh model [16] resulting in the output $s_{m}(\tau)$. This model distinguishes two effects:

- the AM/AM non-linear effect that models amplitude distortions on the input signal;

- the AM/PM non-linear effect that models phase distortions on the input signal.

In this paper, optimization has been accomplished without taking into consideration the AM/PM nonlinear effect due to the HPA (that is known to change the relative position of symbols) since it has been already shown in [22] [23] [24] that this effect can be compensated in case of amplitude and phase modulation, such as wavelet based MRM. Therefore, only the AM/AM non-linear distortion is taken into account through the formula $A(\rho)=\alpha \rho_{n} /[1+\beta] \rho_{n}^{2}$ where $\alpha=2.1587$ and $\beta=1.1517$ are standard values of the constants gathered from the literature [28] and obtained by means of curve-fitting techniques.

Under the above hypothesis, let a discrete periodic (period $\tau$ ) source $S: \forall \tau \rightarrow u(\tau)$ and an analog to digital conversion $A D: \forall u(\tau) \rightarrow \underline{x}(\tau)$ with $\left.\underline{x}(\tau) \in \underline{x}_{k} \mid k=1,2, \ldots 2^{M}\right), \underline{x}_{k} \mid=\left(x_{k}^{(1)}, x_{k}^{(2)}, \ldots x_{k}^{(M)}\right), x_{k}^{(M)}$ being the LSB. $x_{k}^{(i)}$ is then multiplied with the specific weight $w_{i} \in \mathfrak{R}^{+}$of the diagonal matrix $\underline{W}=$ $\operatorname{diag}\left(w_{1}, w_{2}, \ldots w_{M}\right)$. The weighted bit frame $\underline{y}(\tau)=\underline{W} \cdot \underline{x}(\tau)$ is then modulated over the channel opportunities modelled by a LMS channel at Ku-band with zero-mean and power spectral density $\sigma_{L M S}^{2} \in$ $\left\{N_{0}^{B} / 2, N_{0}^{G} / 2\right\}$, with $N_{0}^{B}>N_{0}^{G}$. At the receiver, the distributed vector is $\underline{z}(\tau)=\underline{y}(\tau)+\underline{n}_{r e l}(\tau)$, where $\underline{n}_{r e l}=\left(n_{r e l}^{(1)}, n_{r e l}^{(2)}, \ldots n_{r e l}^{(M)}\right)$ is the relevant noise. Detection is based on Maximum Likelihood (ML) criterion 
and the estimate $\widehat{u}(\tau)$ is obtained by inverse digital to analog (DA) process. The whole system is depicted in figure 3.

\section{WEIGHTS OPTIMIZATION}

Assuming polar binary representation $x_{k}^{(i)}= \pm \sqrt{E_{b}^{(i)}}$, bits in $\underline{x}(\tau)$ can be inverted due to channel impairment and erroneous decisions $\widehat{x}(\tau)$ are possible at the receiver causing a distortion $d(\tau)=$ $[u(\tau)-\hat{u}(\tau)]$. The optimization searches for optimal weights to minimize expected value $E\left[d^{2}(\tau)\right]$. Assuming ergodicity,

$$
E\left\{d^{2}\right\}=\sum_{k=1}^{2^{M}} \sum_{h=1}^{2^{M}} d_{k, h}^{2} P\left(\underline{x}_{k}\right) \cdot P\left(\underline{\hat{x}}_{h} \mid \underline{x}_{k}\right)
$$

where $d_{\zeta, \eta}=u_{\zeta}-\hat{u}_{\eta}, P\left(\underline{x}_{k}\right)$ are the occurrences of $u_{k}$, and $P\left(\underline{x}_{k} \mid \underline{x}_{k}\right)$ the transition probabilities between original and received data. For the independence of the noise samples and the orthogonal properties of wavelets waveforms, $P\left(\underline{\hat{x}}_{k} \mid \underline{x}_{k}\right)=\left[\left(\begin{array}{c}\prod_{i=1}^{M} P_{b}^{(i)} \\ x_{k}^{(i)} \neq x_{h}^{(i)}\end{array}\right) \cdot\left(\begin{array}{c}\prod_{i=1}^{M}\left(1-P_{b}^{(i)}\right) \\ x_{k}^{(i)}=x_{h}^{(i)}\end{array}\right)\right]$ with $P_{b}^{(i)}$ as in (2) and $P_{b a d}$ and $P_{g o o d}$ as in (1). Considering average energy normalized bit frames $E_{b}=1 /{ }_{M} \sum_{i=1}^{M} E_{b}^{(i)}=1, E_{b}^{(i)}=w_{i}^{2} E_{b}$ and

$$
\sum_{i=1}^{M} w_{i}^{2}=M
$$

with $M$ the frame size. In fact, the optimization problem is minimize (3) constrained by (4). The optimization raises $\left(w_{i}>1\right)$ the immunity to noise channel for MSBs, allowing lower robustness $\left(w_{i}<1\right)$ on LSBs, to achieve minimum expected distortion $d(\tau)=[u(\tau)-\widehat{u}(\tau)]$.

Due to the complexity of the above optimization problem which increases with $M$ [10] a solution based on GA has been proposed. In GA a population of candidate solutions called chromosomes and composed by genes evolves toward solutions of an optimization problem. The process is iterative, starts from a population of $L$ randomly generated chromosomes and evolve in generations. At each iteration, the chromosome fitness is evaluated to select candidates with high score from the current population. Then selected candidates are stochastically modified by mutating some gene and/or recombining them among each other, so as to obtain a new population with average higher fitness. In particular, crossover interchanges the elements of two chromosomes with probability $P_{\text {cross }}$, whereas mutation modifies the value of one or more genes within a chromosome with probability $P_{m u t}$. The optimization ends once either the fitness function maintains the same value for $I T_{M A X}$ iterations or the algorithm reaches a selected number of iterations (IT). IT and $I T_{M A X}$ influence the accuracy of GA while the convergence time depends on the size $L$ of the initial population and on the $P_{\text {cross }}$ and $P_{\text {mut }}$ probabilities.

In the actual optimization, described by the flowchart in figure 4, the chromosomes are vectors of $M$ genes $w_{i} \in \mathfrak{R}^{+}$constrained by (4) while the fitness function is as in (3). The evolution process outcome is the chromosome with lowest fitness (i.e., minimum distortion in the received frame).

Figure 5 illustrates selection, crossover and mutation operations: in the given example at $n^{\text {th }}$ iteration $w_{3}$ and $w_{6}$ are discarded due to lowest fitness score, while the rest of the chromosomes are combined to obtain a new set of candidates that can be mutated with $P_{m u t}$. 


\section{TEST AND RESULTS}

In the experiments, a discrete memoryless source $S$ randomly generates integers $u(\tau) \in\{0,255\}$ which are quantized in frames of $M=8$ bits. MRM with $H=-1 / 2$ and $4^{\text {th }}$ order Daubechies wavelets with dilation indices $m=\{10,11,12,13\}$ is deployed. For $m=10$ data are transmitted at $R_{10}=1024 \mathrm{bps}$, which corresponds to a transmission time $T_{10}=0.977 \mathrm{msec}$ per bit, therefore a receiver bandwidth of $B_{10}=$ $2^{(10+1)}=2048 \mathrm{~Hz}$ is necessary to demodulate the signal. For $m=13, R_{13}=8192 \mathrm{bps}$ and $T_{13}=0.122$ msec and $B_{13}=2^{(13+1)}=16384 \mathrm{kHz}$ to receive the signal is needed. Peak Signal to Noise Ratio (PSNR) is used to measure the performance. $P S N R=10 \log _{10}\left(M A X_{I}^{2} / M S E\right)$ where $M A X_{I}^{2}$ is maximum allowable value of $u_{i}$ and $M S E=1 / M_{M} \sum_{i=1}^{M}\left\|u_{i}-\hat{u}_{i}\right\|^{2}$.

At first, to set up the GA evolution we refer to a previous study performed in [16] which analyzed the influence of the GA parameters on the accuracy and convergence performance of the algorithm. Based on [16] only $10 \%$ of a chromosome could vary and a maximum of $40 \%$ of the chromosome parents are allowed to appear on the next generation. Moreover, an initial population of $L=32$ chromosomes was considered, $P_{\text {mut }}=0.3$ and $P_{\text {cross }}=0.5$, whereas $I T_{M A X}=100$ and $I T=1000$. In Table 1 the parameter setting for the experiments is summarized.

Extensive test has been carried out considering various channel opportunities conditions representative of channel occupancy from $40 \%$ to $90 \%$ of the $B \times T$ which reflects a typical CR transmission scenario. Analogously, the parameters of LSM model $\left(P_{\text {good }}, P_{b a d}, A\right)$ have been varied to simulate LSM channel with diverse behavior.

Figure 6 shows weights, which can be obtained from (3) and (4), at varying of $\gamma_{b}$. In this particular case the channel occupancy is $70 \%$ of the $B \times T$. It is worth noting that for very bad channels $\left(\gamma_{b}<-10 d B\right)$ the more significant weights (i.e., $w_{1}, w_{2}, w_{3}, w_{4}$ ) assume higher values with a peak for the MSB's one $w_{1}=2.3$ for $\gamma_{b}=-20 \mathrm{~dB}$. On the other hand, the remaining weights associated to less significant bits are lowered far beyond the unity and the value of the LSB's weight $w_{8}$ is almost negligible. By increasing $\gamma_{b}>5 d B$, weights values tend toward unity because the more the channel conditions improve the less is the sensibility difference between MSB and LSB.

We have compared the proposed system with a benchmark equal power allocation system provided with unequal error protecting (UEP) scheme based on FEC coding. In the latter system, we have deployed ReedSolomon (RS) codes [25] a well-known protection technique currently used in a wide variety of applications, ranging from data storage systems, mobile communications, to satellite communications [26] as well as image [27], audio [28] coding and standards. RS codes are nonbinary cyclic codes with symbols made up of $m$-bit sequences, where $m$ is any positive integer having a value greater than 2. $R S(n, k)$ codes on $m$-bit symbols exist for all $n$ and $k$ for which $0<k<n<2^{n}+2$ where $k$ is the number of data symbols being encoded, and $n$ is the total number of code symbols in the encoded block. The error correcting capability of the generic $R S(n, k)$ code is $t=(n-k) / 2$. UEP is implemented by protecting data with codes with higher or lower code rate $R_{c}^{i}=k^{i} / n^{i}$. At varying the channel error rate, for every carrier an appropriate $R S\left(n^{i}, k^{i}\right)$ code is selected for data protection according to the sensitivity to channel errors of the data carried on. More significant data (e.g., MSB) are protected by codes with higher error correcting capabilities (i.e., higher code rates). In particular, for any average error rate $P_{b}$ the optimization procedure aims at selecting the $m$ codes so that the PSNR is minimized under the bound of constant average code rate $R_{c}=(1 / M) \sum_{i=1}^{M} R_{c}^{i}$.

For our experiments we have selected $m=8$ and $R_{c}=32 / 38=0.84$ which corresponds to an increase of the total bandwidth of about $16 \%$. This was the drawback of using FEC which is by nature a discrete coding scheme. To reduce the complexity of the coding process, we have fixed the number of code symbols in the encoded block $n^{i}=38$. The average error correcting capability of the system is therefore $t=$ $(38-32) / 2=3$ symbols per codeword. In other words, on the average, such a scheme is able to correct up to 3 symbols that contain errors in a codeword. 
In figure 7 the PSNR vs $\gamma_{b}$ is shown for the proposed MRM and for a benchmark MRM with equal power allocation $\left(w_{i}=1\right)$ and UEP based on $R S(38,32)$ as above described. The PSNR trend shows improved quality of the received data. Specifically, the proposed system outperforms the benchmark scheme with a peak gain of $4.1 \mathrm{~dB}$ for $\gamma_{b}=-4 \mathrm{~dB}$. However, for $\gamma_{b}>5 \mathrm{~dB}$ the benefits in using a MUPA approach disappear.

More generally, the prevalence of the proposed unequal power allocation scheme is due to the capability of the optimization procedure to obtain high accuracy by selecting weights in a range of real values. On the other hand, the discrete nature of RS codes, which are constrained to only a definite set of possible code rates, does not guarantee equivalent flexibility in the protection of sensitive data.

In order to assess the correspondence between PSNR and the subjective perception the transmission a standard test grey-level image "Lena" of size $512 \times 512$ coded at 8 bpp has been considered. Figures 8 and 9 show the images received in case of a transmission across a GE channel for $\gamma_{b}=-4 \mathrm{~dB}$. The first image is obtained by adopting equal protection strategy and RS code, whereas the second image is obtained by using the MUPA system. It can be observed that in the first case the image is completely incomprehensible, whilst in the other case it can be easily understood in spite of the errors due to the highly noisy channel.

Figure 10 shows the percentage bandwidth saving achieved by MUPA respect to UEP based on RS coding for target quality (i.e., fixed PSNR) on the transmitted parameters at fixed $\gamma_{b}=-4 \mathrm{~dB}$, for the proposed MRM system. A minimum bandwidth saving above $20 \%$ is noticeable. This is due to the discrete nature of RS codes, which are constrained to only a definite set of possible code rates. On the other hand, MUPA is a continuous process which guarantee more flexibility in the protection of sensitive data.

Moreover, to further confirm the validity of the approach, a 5 seconds from a stereo audio CD signal sampled at $44.1 \mathrm{KHz}$ coded at $16 \mathrm{bps}$ has been considered. For the evaluation of the quality on the received audio signal the perceptual evaluation of audio quality (PEAQ) strategy [29] has been used. PEAQ is technique recommended by the International Telecommunication Union - Telecommunication Standardization Bureau (ITU-T), which evaluates the quality of an audio signal by a single number, called

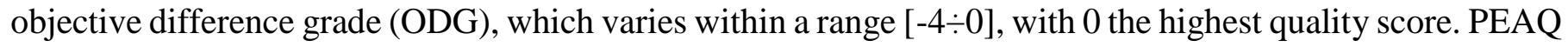
has proven to achieve higher performance than conventional metrics based on MSE on the evaluation of the performance of the conventional audio codecs [29].

Again for $\gamma_{b}=-4 \mathrm{~dB}$, the PEAQ measured on the received audio sequence transmitted with the MUPA proposed scheme is -3.39 , while the benchmark scheme based on RS code achieved a lower score of -3.47. The result achieved is just below the typical performance of low bit rate audio codecs: for the transmission of audio sequences at a rate of $64 \mathrm{Kbit} / \mathrm{s}, \mathrm{MP3} / \mathrm{MPEG}-4$ codecs achieve PEAQ of around -3.36 [30].

\section{CONCLUSIONS AND FUTURE WORKS}

In this work we propose the joint use of unequal power allocation and adaptive multi-rate modulation to increase transmission robustness in CR land mobile satellite (LMS) scenarios where error control coding techniques can be costly to implement due to the uncertainty of the channel parameters $B$ and $T$. Simulation of realistic CR condition provided by the deployment of LMS model at Ku-band, show the efficacy of the proposed system, particularly accentuated for bad channels $\left(\gamma_{b}<5 d B\right)$. The improvement in quality achieved in the parameter domain (i.e., MSE) has been demonstrated against an equally distributed MRM system implementing UEP by means of FEC codes, in similar $B \times T$ constraint. Moreover, transmission of real signals allowed validating the correspondence between quality achieved in the MSE domain and subjective perception. The non-linearity of the satellite channel due to HPA prior to transmission has been considered. Future investigations will aim at evaluating the Total Degradation (TD) for satellite mobile communications where the amplifier nonlinearity can be reduced by operating the HPA at a point with a large output backoff $(\mathrm{OBO})$ rather than in the saturation region. 


\section{Competing interests}

The authors declare that they have no competing interests. 


\section{REFERENCES}

[1] S Scalise, H Ernst, G Harles, Measurement and Modelling of the Land Mobile Satellite Channel at Ku-Band, IEEE Transactions on Vehicular Technology, Vol. 57, No. 2, pp. 693-703, March 2008.

[2] T De Cola, D Tarchi and A Vanelli-Coralli, , Future trends in broadband satellite communications: information centric networks and enabling technologies, International Journal of Satellite Communications and Networking, Volume 33, Issue 5, pages 473-490, September/October 2015.

[3] Q Zhao, BM Sadler, A survey of Dynamic Spectrum Access, IEEE Signal Processing Magazine, 24(3), 79-89 (2007).

[4] M Fadda, V Popescu, M Murroni, P Angueira, J Morgade, On the feasibility of unlicensed communications in the TV white space: Field measurements in the UHF band (2015) International Journal of Digital Multimedia Broadcasting, 2015, art. no. 319387.

[5] M J Abdel-Rahman, M Krunz, and R Erwin Exploiting cognitive radios for reliable satellite communications, International Journal of Satellite Communications and Networking, Volume 33, Issue 3, pages 197-216, May/June 2015

[6] M Murroni, PR Venkatesha, PJ Marques, B Bochow, D Noguet, C Sun, K Moessner and H Harada, IEEE 1900.6 Spectrum Sensing Interfaces and Data Structures for Dynamic Spectrum Access and other Advanced Radio Communication Systems Standard: Technical Aspects and Future Outlook, IEEE Comm. Magazine, 49(12), 118127 (2011).

[7] L Safatly, B Aziz, A Nafkha, Y Louet, Y Nasser, A El-Hajj, KY Kabalan, Blind spectrum sensing using symmetry property of cyclic autocorrelation function: from theory to practice. EURASIP Journal on Wireless Communications and Networking 2014, 2014:26 doi:10.1186/1687-1499-2014-26.

[8] M Jia, X Liu, X Gu and Q Guo, Joint cooperative spectrum sensing and channel selection optimization for satellite communication systems based on cognitive radio, International Journal of Satellite Communications and Networking, Article first published online: 16 DEC 2015, DOI: 10.1002/sat.1169.

[9] M Sanna, M Murroni, Nonconvex optimization of collaborative multiband spectrum sensing for cognitive radios with genetic algorithms, International Journal of Digital Multimedia Broadcasting (2010). doi: $10.1155 / 2010 / 531857$.

[10] GW Wornell and AV Oppenheim, Wavelet-based representations for a class of self-similar signals with application to fractal modulation, IEEE Trans. Inform. Theory, 38, 785-800 (1992).

[11] L Atzori, DD Giusto, M Murroni Performance analysis of fractal modulation transmission over fast-fading wireless channels, IEEE Trans. Broadcasting, 48, 103-110, (2002).

[12] T Bruggen and P Vary, "Unequal Error Protection by Modulation with Unequal Power Allocation," IEEE Communication Letters, 9(6), 484- 486 (2005).

[13] M Murroni Performance analysis of modulation with unequal power allocations over fading channels: A genetic algorithm approach, EW2008 - 14th European Wireless Conference 2008, Prague, Czech Republic; 22 - 25 June 2008.

[14] M Lixia, M Murroni, Adaptive Wavelet Modulation with Unequal Power Allocation for DSA Networks Proceedings of the IEEE International Conference on Communications, ICC 2010, Cape Town, 23-27 May 2010.

[15] E Lutz, D Cygan, M Dippold, F Dolainsky, and W Papke, The land mobile satellite communication channelRecording, statistics, and channel model, IEEE Transactionon on Vehicular. Technology., vol. 40, pp. 375-384, May 1991.

[16] A A M Saleh, Frequency-Independent and Frequency-Dependent Nonlinear Models of TWT Amplifiers IEEE Transactions on Communications, 29 (11), pp. 1715-1720 (1981).

[17] D Whitley, A genetic algorithm tutorial. Statistics and Computing, 4, 65-85 (1994).

[18] M Lixia, M Murroni, Peak-to-average power ratio reduction in multi-carrier system using genetic algorithms, IET Signal Processing, 5(3), 356-363, (2011).

[19] M Sanna, M Murroni. "Nonconvex Optimization of Collaborative Multiband Spectrum Sensing for Cognitive Radios with Genetic Algorithms". International Journal of Digital Multimedia Broadcasting, art. no. 531857 (2010).

[20] M Fadda, M. Murroni, C Perra, V Popescu TV White Spaces Exploitation for Multimedia Signal Distribution Signal Processing: Image Communication, 27(8), 893-899 (2012). 
[21] M Sanna, M Murroni Opportunistic wideband spectrum sensing for cognitive radios with genetic optimization 2010 IEEE International Conference on Communications, ICC 2010; Cape Town; South Africa; 23 -27 May 2010

[22] R De Gaudenzi, A Guillen, I Fabregas, A, Martinez, "Performance Analysis of Turbo-Coded APSK Modulations over Nonlinear Satellite Channels", IEEE Trans. Wireless Commun., 5 (9), 2396-2407, (2006).

[23] M Anedda, A Meloni, M Murroni 64-APSK constellation and mapping optimization for satellite broadcasting using genetic algorithms IEEE Transactions on Broadcasting 62 (1), 1-9, (2016),

[24] WZ Zhong, Q Guo, Y Guo Performance evaluation of wavelet packet modulation over mobile satellite channel Information Technology Journal, 8 (3), pp. 310-317 (2009).

[25] T H Hu and M H Chang, List Decoding of Generalized Reed-Solomon Codes by Using a Modified Extended Key Equation Algorithm EURASIP Journal on Wireless Communications and Networking, January 2011, 2011: 212136. doi:10.1155/2011/212136.

[26] X Youshi and T Zhang, Variable shortened-and-punctured Reed-Solomon codes for packet loss protection, IEEE Trans. on Broadcasting, 48 (3), 237-245, (2002).

[27] P N Huu, V T Quang, T Miyoshi, Multi-hop Reed-Solomon encoding scheme for image transmission on wireless sensor networks, IEEE International Conference on Communications and Electronics, ICCE 2012, Hue, 1-2 August 2012.

[28] A Khalifeh and H Yousefi'zadeh, Optimal Audio Transmission Over Error-Prone Wireless Links. IEEE Trans. on Multimedia, 12 (3), 204-214, (2010).

[29] Recommendation ITU-R BS.1387-1, 11/01, Method for Objective Measurements of Perceived Audio Quality (PEAQ).

[30] M Salovarda, I Bolkovac and H Domitrovic, "Estimating perceptual audio systems quality using PEAQ algorithm," 18th International Conference on Applied Electromagnetics and Communications, Dubrovnik, 12-14 October 2005. 


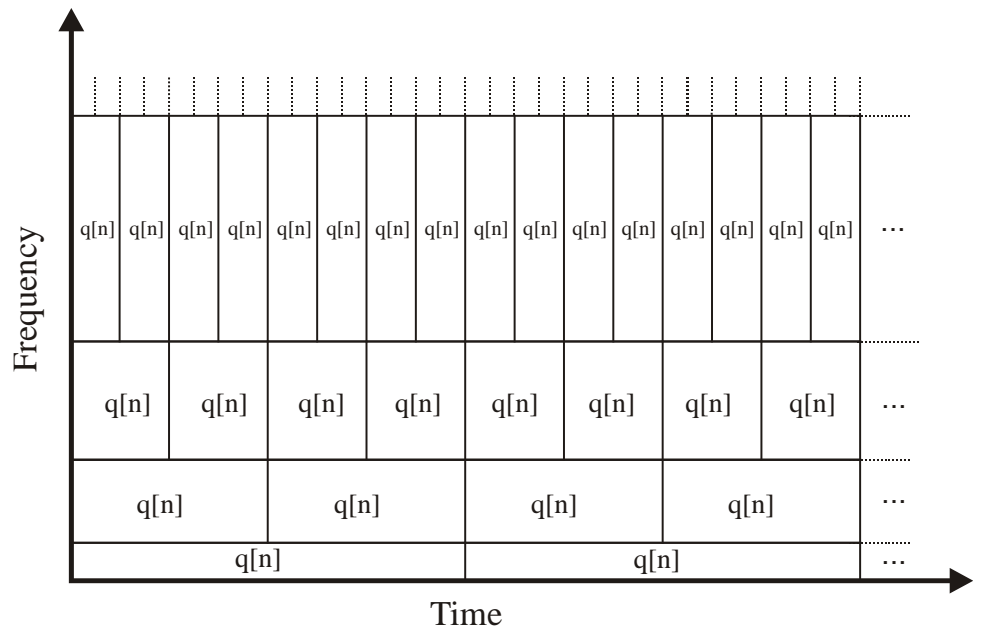

Figure. 1. Time-Frequency portrait for MRM with $H=1 / 2$

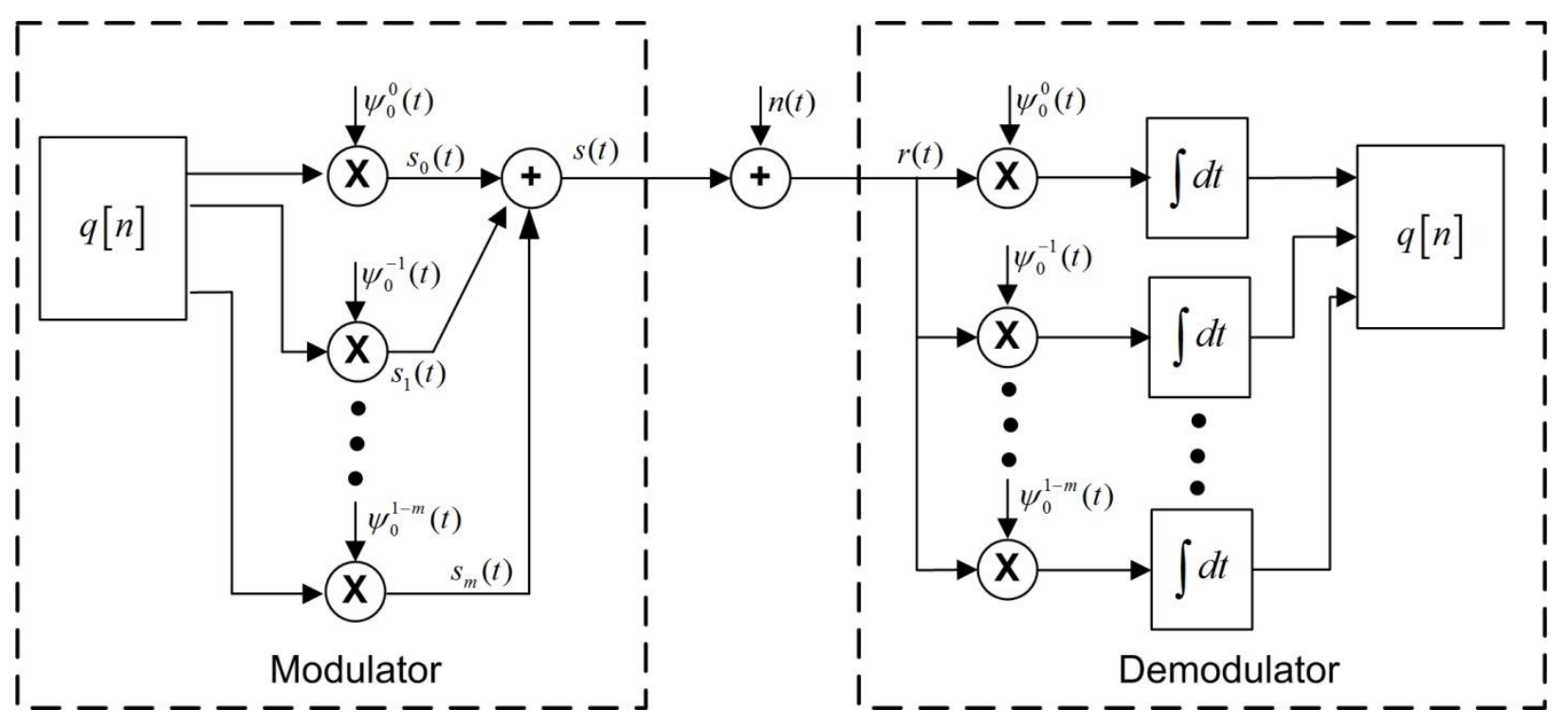

Figure. 2. MRM Modulator and Demodulator

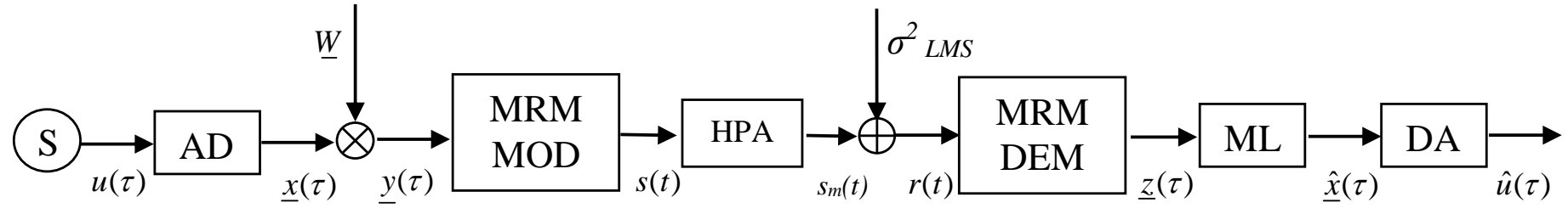

Figure. 3. System model 


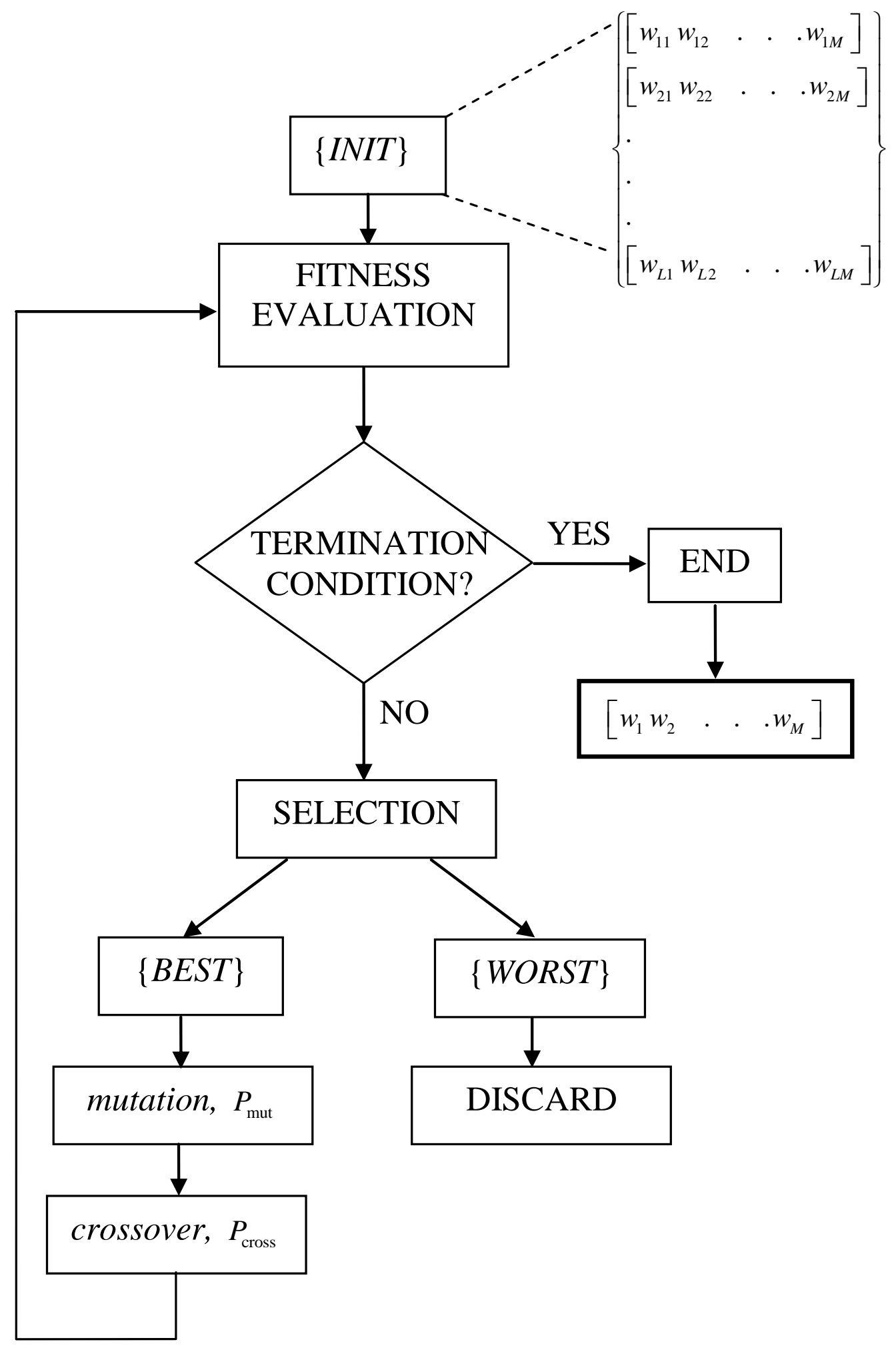

Figure. 4. Flowchart of the proposed GA 


\section{Generation n}

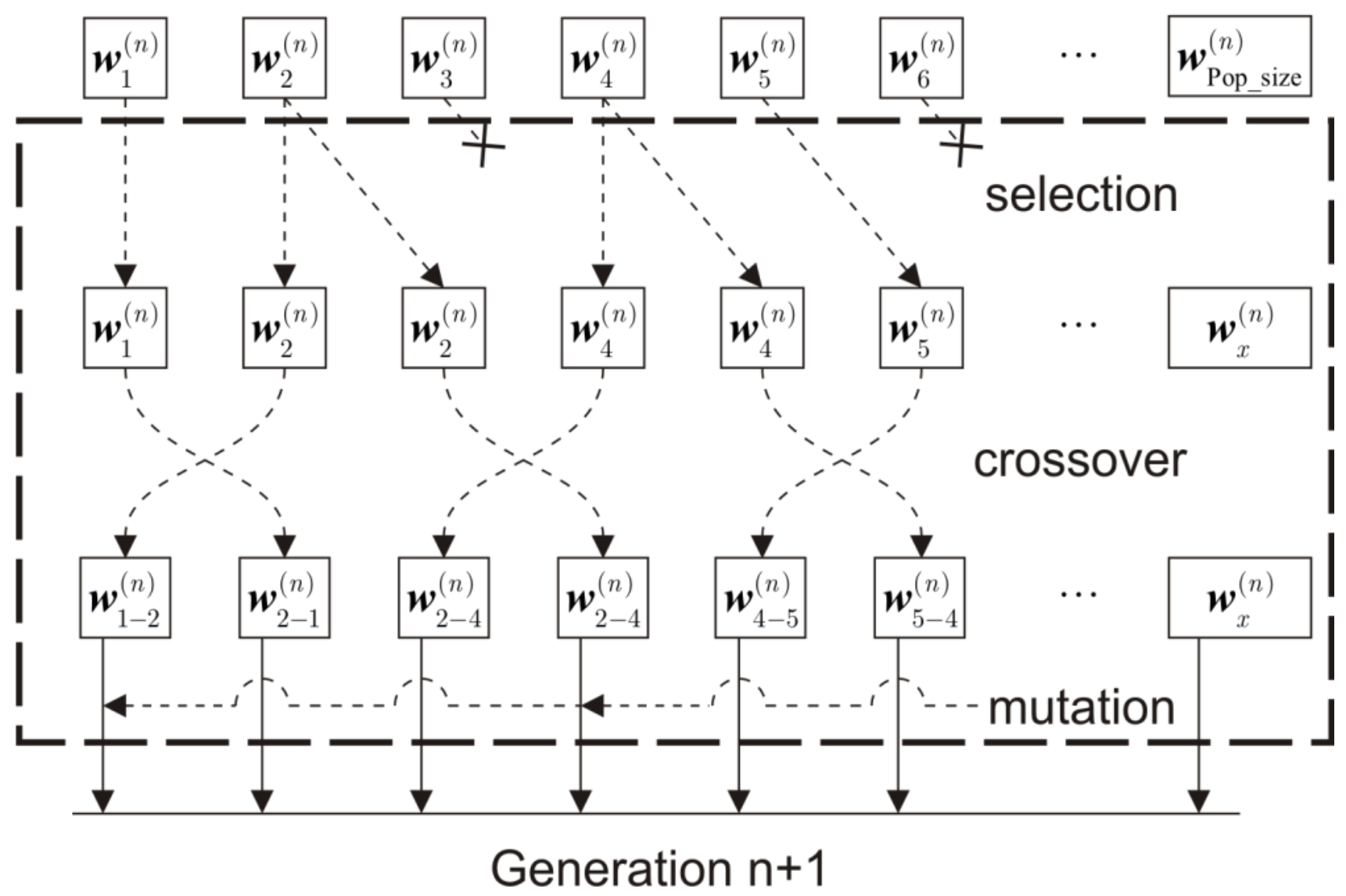

Figure. 5. Example for selection, crossover and mutation operators

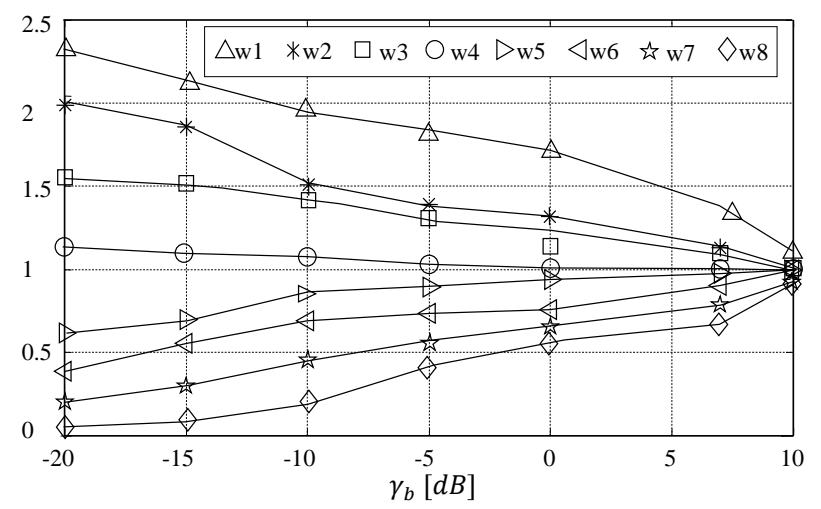

Figure 6. Weights values vs. $\gamma_{b}$ 


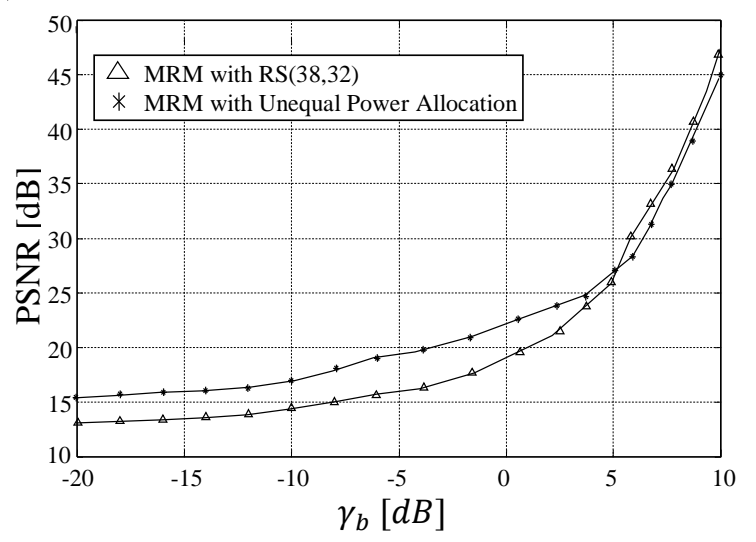

Figure 7. PSNR vs. $\gamma_{b}$

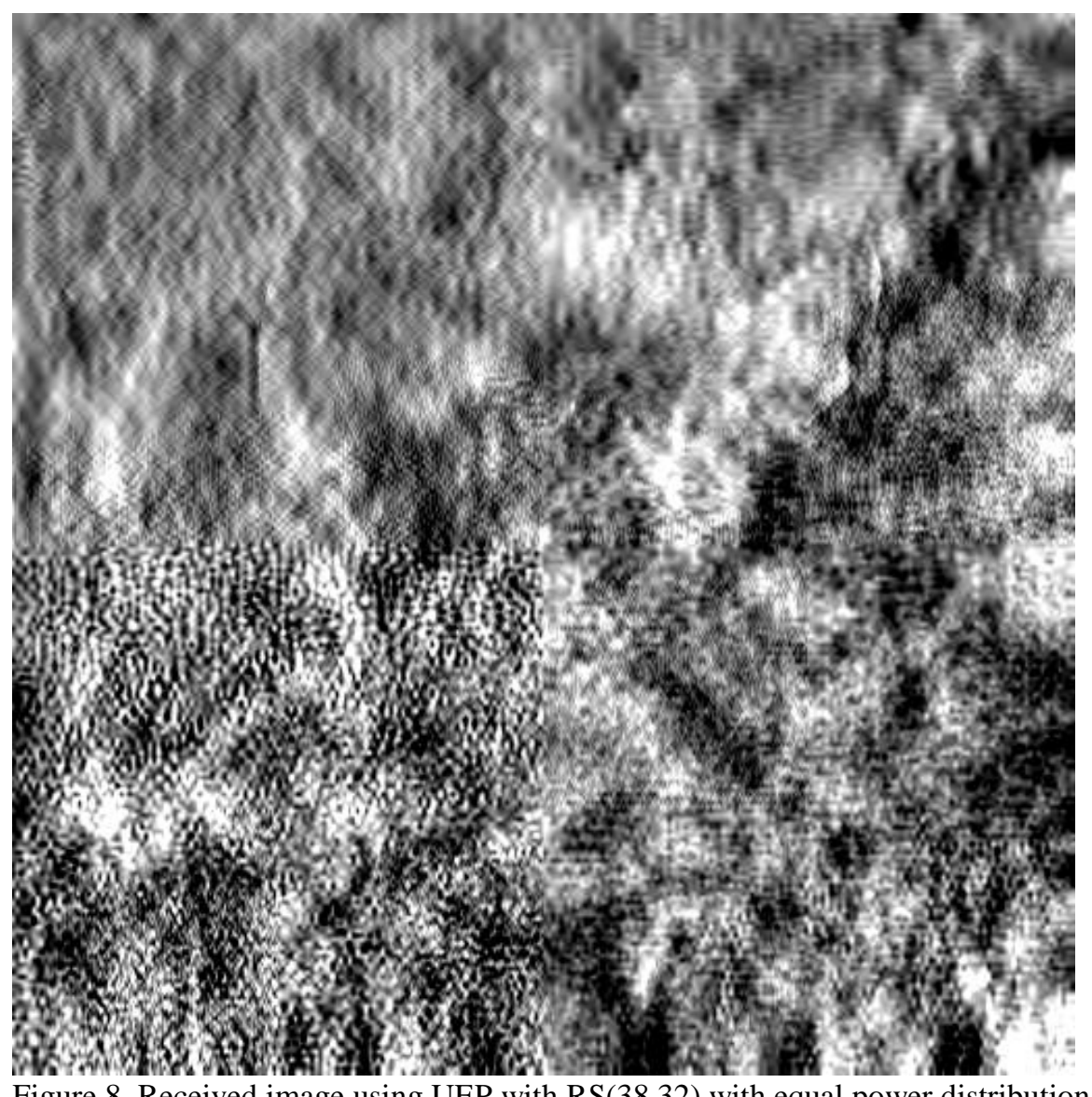

Figure 8. Received image using UEP with RS $(38,32)$ with equal power distribution 


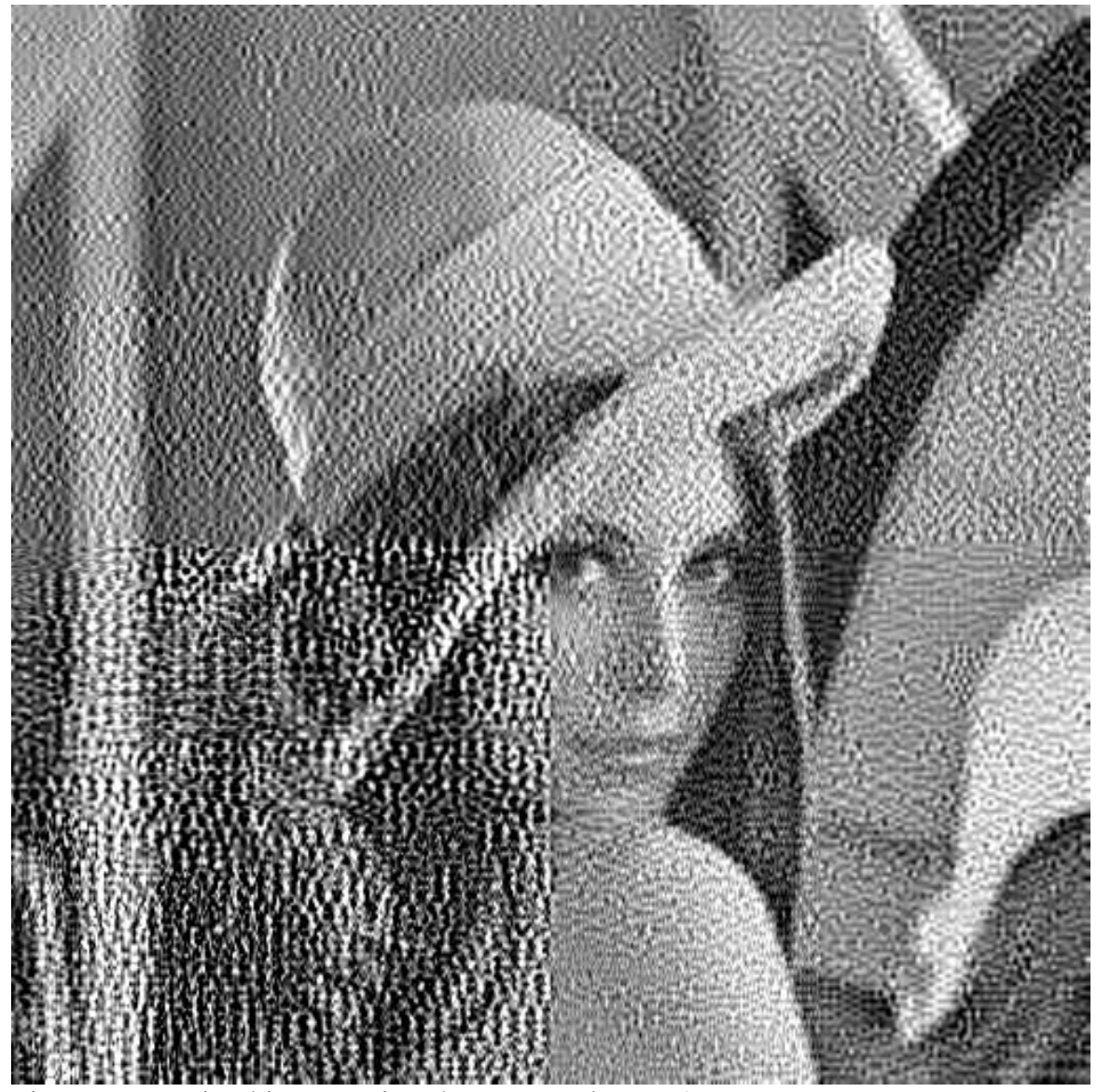

Figure 9. Received image using the proposed MUPA

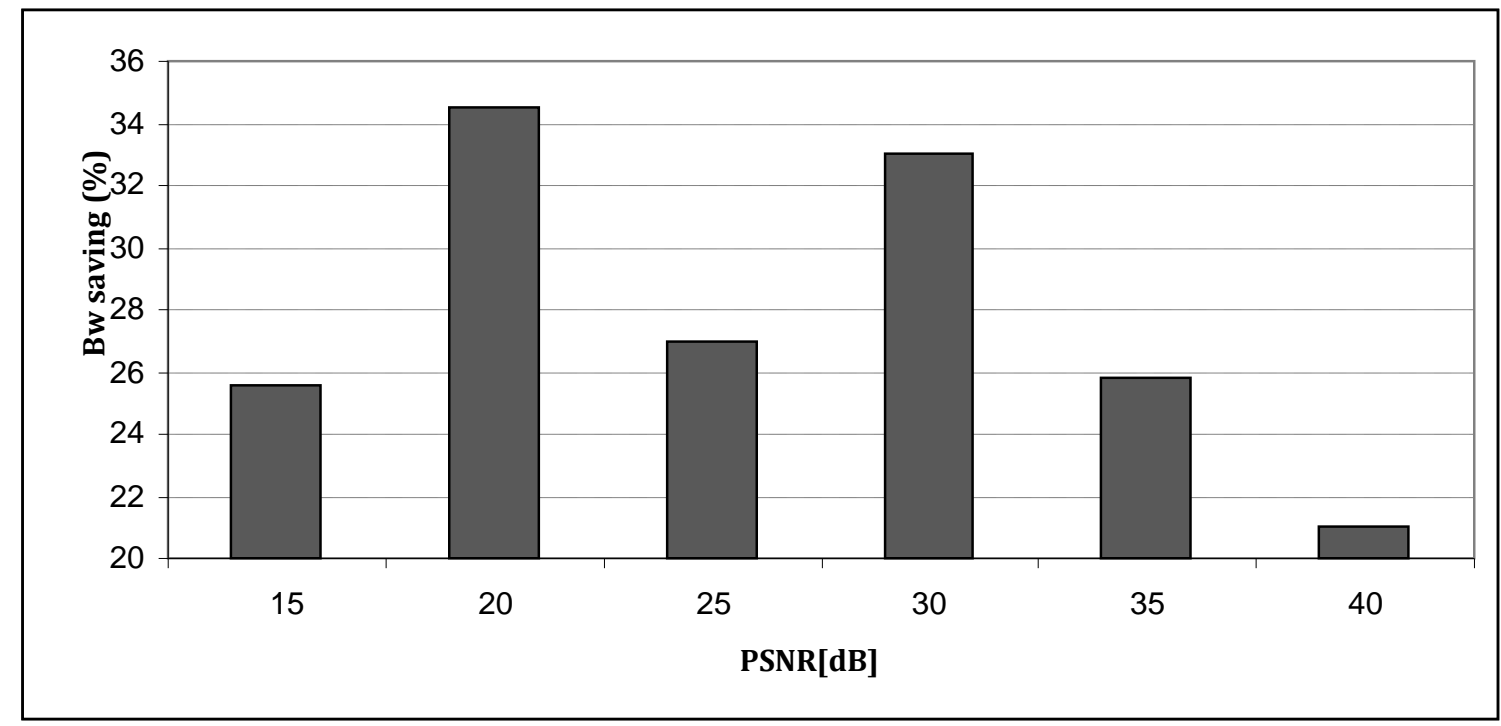

Figure 10. Percentage bandwidth saving for fixed quality on the transmitted parameter achieved by MUPA against UEP by RS codes for the MRM scheme 
TABLE 1: PARAMETERS SETTING FOR EXPERIMENTS

\begin{tabular}{lll}
\hline \hline Symbol & Definition & Setting \\
\hline$S$ & source & discrete, memoryless \\
$A D / D A$ & analog to digital/digital to analog & uniform quantizer, natural binary mapping \\
$M$ & processes & 8 \\
$L$ & bit frame size (chromosome size) & 32 \\
$w_{i}$ & size of initial population & $\in \mathfrak{R}^{+}$ \\
$P_{\text {mut }}$ & weight (gene) & 0.3 \\
$P_{\text {cross }}$ & mutation probability & 0.5 \\
$I T$ & crossover probability & 1000 \\
$I T_{M A X}$ & number of iteration & maximum number of iteration with \\
& unchanged fitness &
\end{tabular}

